\title{
Development and Validation of LAMP Primer Sets for Rapid Identification of Aspergillus Fumigatus Carrying the cyp51A TR46 Azole Resistance Gene
}

Plinio Trabasso ( $\nabla$ trabasso@unicamp.br)

State University of Campinas

Tetsuhiro Matsuzawa

University of Nagasaki

Teppei Arai

Chiba University

Daisuke Hagiwara

University of Tsukuba

Yuzuru Mikami

Chiba University

Maria Luiza Moretti

State University of Campinas

Akira Watanabe

Chiba University

\section{Research Article}

Keywords: rapid identification, new LAMP assay method, azole resistance, cyp51A gene, Aspergillus fumigatus

Posted Date: May 13th, 2021

DOl: https://doi.org/10.21203/rs.3.rs-516075/v1

License: (a) (1) This work is licensed under a Creative Commons Attribution 4.0 International License. Read Full License 


\section{Development and validation of LAMP primer sets for rapid identification of}

Aspergillus fumigatus carrying the cyp51A TR 46 azole resistance gene

Plinio Trabasso ${ }^{1^{*}}$, Tetsuhiro Matsuzawa ${ }^{2}$, Teppei $\mathrm{Arai}^{3}$, Daisuke Hagiwara ${ }^{3}, 4,5$, Yuzuru Mikami $^{3}$, Maria Luiza Moretti ${ }^{1}$, Akira Watanabe ${ }^{3}$

School of Medical Sciences, University of Campinas, Campinas, Sao Paulo, Brazil ${ }^{1}$ University of Nagasaki, Nagasaki, Japan²

Medical Mycology Research Center, Chiba University, Chiba, Japan ${ }^{3}$

Faculty of Life and Environmental Sciences, University of Tsukuba, Tsukuba, Ibaraki, Japan ${ }^{4}$ Microbiology Research Center for Sustainability, University of Tsukuba, Ibaraki, Japan ${ }^{5}$

*Corresponding author: Plinio Trabasso, Dept of Internal Medicine, School of Medical Sciences, State University of Campinas, Rua Tessalia Vieira de Camargo, 126, Campinas, Sao Paulo, Brazil.

Phone: +55 (19) 3521-7451, Fax: +55 (19) 3521-7054, e-mail: trabasso@unicamp.br ORCID: 0000-0002-0588-4859 (Plinio Trabasso)

\section{ABSTRACT}

Infections due to triazole resistant Aspergillus fumigatus are increasingly reported worldwide and are associated with treatment failure and mortality. The principal class of azole resistant isolates is characterized by the presence of tandem repeats of $34 \mathrm{bp}$ or 46 bp within the promoter region of the cyp51A gene. Loop-mediated isothermal amplification (LAMP) is a widely used nucleic acid amplification system which is fast and specific. Here 
we describe a LAMP assay method to detect the $46 \mathrm{bp}$ tandem repeat insertion in the cyp51A gene promoter region based on novel LAMP primer sets. It also differentiated strains with $\mathrm{TR}_{46}$ tandem repeats from those with $\mathrm{TR}_{34}$ tandem repeats. These results showed this TR R6 $_{4}$-LAMP method is specific, rapid, and also provides crucial insights to enable development of novel antifungal therapeutic strategies against severe fungal infections due to $A$. fumigatus with $\mathrm{TR}_{46}$ tandem repeats.

KEYWORDS: rapid identification, new LAMP assay method, azole resistance, cyp51A gene, Aspergillus fumigatus

\section{Introduction}

Antimicrobial resistance was defined by the World Health Organization (WHO) as one of the most important threats to human health, since it can compromise our ability to treat infectious diseases, as well as undermining other advances in health care ${ }^{1}$. Although bacterial resistance still remains the most common finding in the clinical setting, fungal resistance, specially to azole antifungals among filamentous fungi, is relentlessly increasing worldwide ${ }^{2,3,4}$. Resistance to azole drugs can be due, in general, to two major genetic mechanisms; point mutation(s) in the cyp51A open reading frame with or without tandem repeat (TR) of 34 or 46 base pair (bp) in the promoter region of the gene $\left(\mathrm{TR}_{34}\right.$ or $\left.\mathrm{TR}_{46}\right)$ and overexpression of oligonucelotide sequence in the cyp51A gene ${ }^{5}$. These mutations and overexpression of the gene confer different levels of resistance ${ }^{6}$. Point mutations are the consequence of previous exposure to azole drugs in a clinical setting, such as for prophylaxis or therapeutic purposes ${ }^{7,8}$. On the other hand, TR with point mutation(s) are the aftermath of previous exposure to azole fungicides. In an agricultural setting, azole fungicides are widely 
used to prevent fungal contamination in a large variety of crop and plant protection, which may allow the TR type resistant strains to be emerged in the environments and the conidia to disperse to the air ${ }^{8,9}$. In Brazil, agribusiness represents about $25 \%$ of the Brazilian Gross National Product (GNP) (http://www.agricultura.gov.br), and in this scenario, fungicide use has steadily increasing over the years. The consumption of pesticides in Brazil increased 190\% in 2010 and fungicides corresponded to $14 \%$ of this (https://www.ipessp.edu.br).

In the Netherlands, prevalence of TR type resistant strains was high. Of 952 clinical $A$. fumigatus strains were collected and found to include 225 and 98 had $\mathrm{TR}_{34}$ and $\mathrm{TR}_{46}$, respectively ${ }^{10}$. Another study revealed that $13 \mathrm{TR}_{34}$ strains and $3 \mathrm{TR}_{46}$ strains were among 105 clinical strains ${ }^{10}$. Additionally, there are number of reports of fatal invasive aspergillosis caused by $A$. fumigatus carrying the $\mathrm{TR}_{46}$ in an acute myeloid leukemia (AML) patients and hematopoietic stem cell transplant recipients ${ }^{11}$. Higher mortality of patients with invasive aspergillosis caused by azole resistant strain has been reported ${ }^{11,12}$. Thus, a rapid and specific method to identify the presence of TR would contribute to faster therapeutic decision making ${ }^{13}$.

As one of the promising diagnostic tools for the azole resistant $A$. fumigatus, loop-mediated isothermal amplification (LAMP) for the development of improved DNA-based diagnostic kits has been reported ${ }^{14}$. In general, the LAMP method was found to be similar or superior to the general PCR method, and more specific, lower-cost and simpler. LAMP based approaches have been applied to a wide range of samples, such as whole blood, paraffin-embedded tissues, and various microbial pathogens. In this paper, we report a novel LAMP assay method which selectively detects triazole resistant $A$. fumigatus strains due to presence of double $\mathrm{TR} 46\left(\mathrm{TR}_{46}{ }^{2}\right)$ or triple $\mathrm{TR}_{46}\left(\mathrm{TR}_{46}{ }^{3}\right)$ in the cyp51A promoter region. 


\section{Results}

Antifungal susceptibility tests

Drug susceptibilities of $41 \mathrm{~A}$. fumigatus strains against azole drugs itraconazole and voriconazole are shown in Table 2 . Thirty strains designated as wild type were isolates from clinical specimens, and they were confirmed to be susceptible to itraconazole and voriconazole. The remaining 11 strains ( $\mathrm{TR}_{34}$ and $\left.\mathrm{TR}_{46}\right)$ were resistant to voriconazole, and most of them showed MIC values of $>8 \mu \mathrm{g} / \mathrm{ml}$ against voriconazole. Among the 11 strains, 2 strains (IFM64460 with TR34/L978H, and IFM64733 with TR34/LH98H) were resistant to itraconazole, and remaining 9 strains were susceptible to itraconazole (Table 1).

Table 1. Aspergillus fumigatus strains used in this experiment and their drug susceptibility profiles against itraconazole and voriconazole.

\begin{tabular}{|c|c|c|c|c|}
\hline \multirow{2}{*}{$\begin{array}{l}\text { Strain } \\
\text { No. }\end{array}$} & \multirow{2}{*}{ Isolation sources } & \multirow{2}{*}{ cyp51A genotypes } & \multicolumn{2}{|c|}{$\begin{array}{l}\text { MIC values } \\
(\mu \mathrm{g} / \mathrm{ml})\end{array}$} \\
\hline & & & ITCZ & VRCZ \\
\hline IFM63432 & clinic & $\mathrm{TR}_{46}{ }^{2} \mathrm{Y} 121 \mathrm{~F} / \mathrm{T} 289 \mathrm{~A}$ & 4 & $>8$ \\
\hline BE1-2 & environment (bulb) ${ }^{a}$ & $\mathrm{TR}_{46}{ }^{2} \mathrm{Y} 121 \mathrm{~F} / \mathrm{T} 289 \mathrm{~A}$ & 2 & $>8$ \\
\hline BE1-4 & environment (bulb) & $\mathrm{TR}_{46^{2}}{ }^{2} \mathrm{Y} 121 \mathrm{~F} / \mathrm{S} 363 \mathrm{P} / \mathrm{I} 364 \mathrm{~V} / \mathrm{G} 448 \mathrm{~S}$ & 2 & $>8$ \\
\hline BE 3-5 & environment (bulb) & $\mathrm{TR}_{46}{ }^{2} \mathrm{Y} 121 \mathrm{~F} / \mathrm{T} 289 \mathrm{~A}$ & 2 & $>8$ \\
\hline BE 3-6 & environment (bulb) & $\mathrm{TR}_{46}{ }^{2} \mathrm{Y} 121 \mathrm{~F} / \mathrm{T} 289 \mathrm{~A}$ & 2 & $>8$ \\
\hline BE 1-1 & environment (bulb) & $\mathrm{TR}_{46}{ }^{3} / \mathrm{Y} 121 \mathrm{~F} / \mathrm{M} 172 \mathrm{I} / \mathrm{T} 289 \mathrm{~A} / \mathrm{G} 448 \mathrm{~S}$ & 2 & $>8$ \\
\hline W1-4 & environment (bulb) & $\mathrm{TR}_{46}{ }^{3} / \mathrm{Y} 121 \mathrm{~F} / \mathrm{M} 172 \mathrm{I} / \mathrm{T} 289 \mathrm{~A} / \mathrm{G} 448 \mathrm{~S}$ & 2 & $>8$ \\
\hline W2-12-1 & environment (bulb) & $\mathrm{TR}_{46}{ }^{3} / \mathrm{Y} 121 \mathrm{~F} / \mathrm{M} 172 \mathrm{I} / \mathrm{T} 289 \mathrm{~A} / \mathrm{G} 448 \mathrm{~S}$ & 2 & $>8$ \\
\hline IFM64460 & clinic & $\mathrm{TR}_{34} / \mathrm{L} 98 \mathrm{H}$ & $>8$ & $>8$ \\
\hline IFM64733 & environment & $\mathrm{TR}_{34} / \mathrm{L} 98 \mathrm{H}$ & $>8$ & $>8$ \\
\hline $3-1-B$ & environment (bulb) & $\mathrm{TR}_{34} / \mathrm{L} 98 \mathrm{H} / \mathrm{T} 289 \mathrm{~A} / \mathrm{l} 364 \mathrm{~V} / \mathrm{G} 448 \mathrm{~S}$ & 2 & $>8$ \\
\hline IFM62918 & clinic & wild & 0.5 & 1 \\
\hline IFM62799 & clinic & wild & 0.5 & 1 \\
\hline IFM60516 & clinic & wild & 1 & 1 \\
\hline
\end{tabular}




\begin{tabular}{|c|c|c|c|c|}
\hline IFM58402 & clinic & wild & 0.5 & 0.5 \\
\hline IFM51977 & clinic & wild & 0.25 & 0.25 \\
\hline IFM60065 & clinic & wild & 1 & 0.5 \\
\hline IFM61960 & clinic & wild & 0.5 & 0.5 \\
\hline IFM51748 & clinic & wild & 0.125 & 0.125 \\
\hline IFM63666 & clinic & wild & 1 & 2 \\
\hline IFM62520 & clinic & wild & 1 & 0.5 \\
\hline IFM50999 & clinic & wild & 0.5 & 0.5 \\
\hline IFM50268 & clinic & wild & 0.25 & 0.125 \\
\hline IFM55548 & clinic & wild & 0.25 & 0.25 \\
\hline IFM63311 & clinic & wild & 1 & 0.5 \\
\hline IFM63355 & clinic & wild & 2 & 2 \\
\hline IFM60901 & clinic & wild & 0.5 & 0.5 \\
\hline IFM62674 & clinic & wild & 1 & 2 \\
\hline IFM62709 & clinic & wild & 0.5 & 0.5 \\
\hline IFM52659 & clinic & wild & 1 & 1 \\
\hline IFM57130 & clinic & wild & 0.25 & 0.125 \\
\hline IFM60814 & clinic & wild & 0.5 & 0.5 \\
\hline IFM49435 & clinic & wild & 0.25 & 0.25 \\
\hline IFM61572 & clinic & wild & 0.5 & 0.5 \\
\hline IFM50669 & clinic & wild & 0.5 & 0.25 \\
\hline IFM59832 & clinic & wild & 0.5 & 0.5 \\
\hline IFM55044 & clinic & wild & 0.25 & 0.25 \\
\hline IFM47670 & clinic & wild & 0.5 & 0.5 \\
\hline IFM51978 & clinic & wild & 0.5 & 0.25 \\
\hline IFM58328 & clinic & wild & 0.5 & 0.5 \\
\hline IFM60369 & clinic & wild & 0.5 & 0.5 \\
\hline
\end{tabular}

a: obtained from plant bulbs. 


\section{Primer design}

The most important step in LAMP assay is the design of primers. In LAMP assay, 6 primers are necessary to amplify the targeted region under isothermal condition. First, we inspected the promoter region (-461 bp to $-296 \mathrm{bp}$ counted from start codon) of the cyp51A gene to select a set of primer sequences that specifically amplify the repeated 46 bp sequence in strains with a TR 46 mutation (Fig. 1 and 2). To enable for specific amplification against repeated $\mathrm{TR}_{46}$ sequences, $\mathrm{B} 2$ was set on the joint of two $46 \mathrm{bp}$ sequences. To obtain a specific and rapid LAMP primer set in the LAMP assay another 5 sequences for primer sets were chosen in the target region according to the standard criteria. Namely 6 primers (F1, F2, $\mathrm{F} 3, \mathrm{~B} 1, \mathrm{~B} 2, \mathrm{~B} 3)$ that target 6 specific regions of a DNA template of the TR 46 gene of cyp51A were selected, and in addition 2 loop primers (LF, LB) were also chosen to accelerate the reaction (Fig. 1). Based on the above several information, several new candidates of LAMP primers were designed and their utility tested. From those, one useful LAMP primer sets based on the detection of $\mathrm{TR}_{46}$ regions in cyp51A gene was selected (Table 2).

Fig. 1

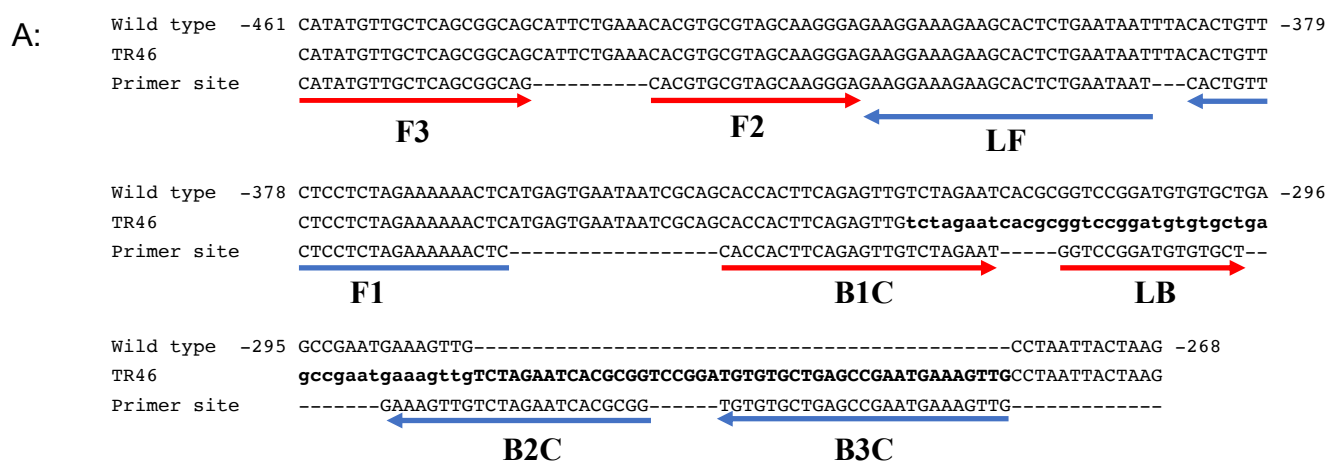

B:

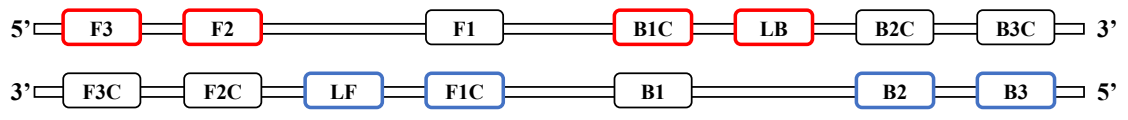


Fig. 1. Genetic information for the design of the LAMP primer sets.

A: Schematic illustration of cyp51A gene showing LAMP primer positions and corresponding sequences of TR46 bp promoter tandem repeat in comparion with those of wild type.

B: Primers F3, F2, F1, B1, B2 and B3 show primer sequence positions. Sequences of some primers

are complementary as shown in Table 2. See LAMP primer and methods which are shown in the refereces 19 and 20 .

Fig. 2

A:

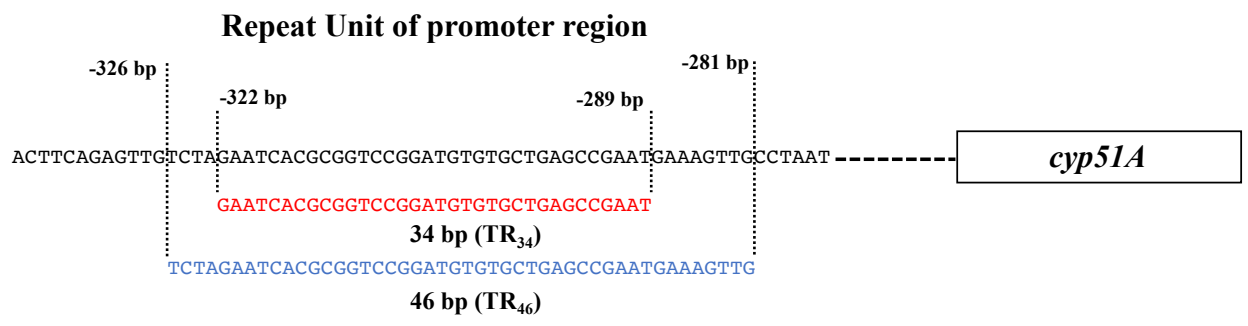

$B:$

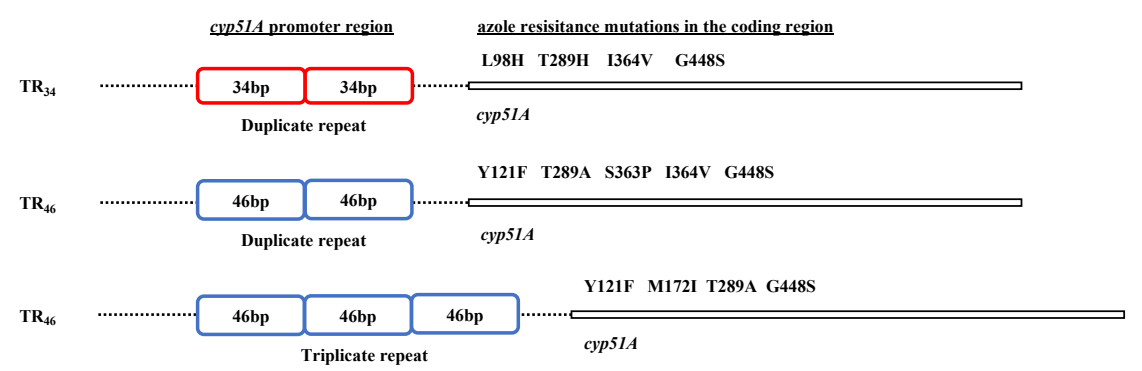

Fig. 2. Illustration of tandem repeat regions of cyp51A genes used in this experiment.

A: Tandem repeat unit of promoter genes of $\mathrm{TR}_{34}$ and $\mathrm{TR}_{46}$.

B: Tandem repeat: $34 \mathrm{bp}$ (double) and $46 \mathrm{bp}$ (double or triple), and cyp51A gene associated point mutation place. 
Table 2. Sequence information of newly designed TR46-LAMP primer sets in the present experiment.

\begin{tabular}{ll}
\hline LAMP primer names & Sequence (5' to 3') \\
\hline F3 & CATATGTTGCTCAGCGGCAG \\
B3 & CAACTTTCATTCGGCTCAGCA \\
& (complementary sequence) \\
FIP (F1 complementary+ F2) & GAGTTTTTTCTAGAGGAGAACAGTG- \\
& CACGTGCGTAGCAAGGGA \\
& (F1: complementary sequence) \\
BIP (B1 + B2 complementary) & CACCACTTCAGAGTTGTCTAGAAT- \\
& ACCGCGTGATTCTAGACAACTTTC \\
& (B1: Complementary) \\
LF & ATTATTCAGAGTGCTTCTTTCCTTC \\
LB & (complementary sequence) \\
\hline
\end{tabular}

Validation of LAMP primer sets for $\mathrm{TR}_{46}$

Specificity of the primer sets was tested using various types of $A$. fumigatus strains, such as wild isolates, and environmental or clinical azole resistance isolates (Fig. 3). In this study, IFM 63432 strain was used as a positive control. As shown in Fig. 3A-i, TR46 LAMP primer could not amplify the DNA from 30 strains of azole drug susceptible clinical isolates of $A$. fumigatus. However, the start of the LAMP amplification in the control strain of $A$. fumigatus strains (IFM 63432) was at around 50 min. On the other hands, TR 46 LAMP primer could amplify DNA from A. fumigatus strains carrying the duplicate $46 \mathrm{bp}$ promoter repeat in cyp51A gene 
(IFM63432, Be1-2, BE1-4, BE3-5, BE3-6) as shown in Fig. 3A-ii. This result suggests that the present LAMP primer could amplify the four $\mathrm{TR}_{46}{ }^{2}$ strains harboring the TR46 resistant mutation (TR46/Y121F/T289A). It was also confirmed that TR 46 LAMP primer could amplify DNA of $A$. fumigatus strains carrying three $\mathrm{TR}_{46}{ }^{3}$ strains (BE1-!, W1-4, W2-12-1) (Fig.3A-iii). When this $\mathrm{TR}_{46}$ LAMP primer was tesed for three $\mathrm{TR}_{34}{ }^{2}$ strains (Table 1 ), namely strains IFM64460 and 64733 (with mutation of TR34/L98H) and strain 3-1-B (with mutations of $\mathrm{TR}_{34} / \mathrm{L} 98 \mathrm{H} / \mathrm{Y} 289 / \mathrm{T} 289 \mathrm{~A} / \mathrm{I364V/G448S),} \mathrm{DNA} \mathrm{amplification} \mathrm{was} \mathrm{not} \mathrm{observed} \mathrm{(Fig.} \mathrm{3B-i} \mathrm{and}$ B-ii). These results also suggested that the present LAMP primer could not detect $\mathrm{TR}_{34}{ }^{2} \mathrm{drug}$ resistant strains regardless of their point mutation site in cyp51A gene (Fig. 3 B-i and B-ii). These studies confirmed that newly established TR TR $_{46}$ LAMP primer set was specific $A$. fumigatus strains with TR of double or triple 46-bp promoter tandem repeats in cyp51A gene.

Fig. 3

A

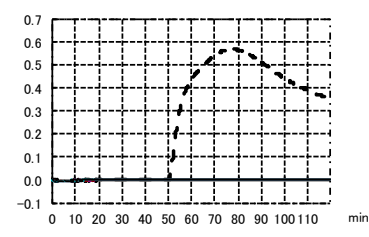

B

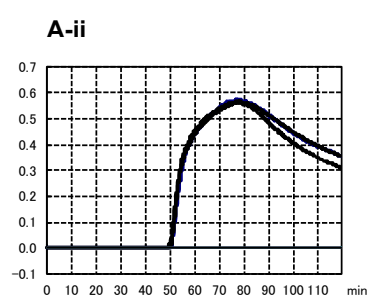

B-i

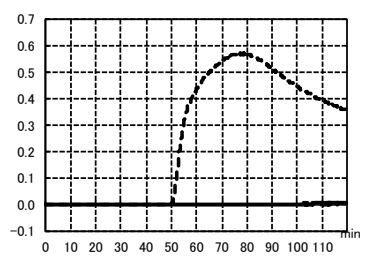

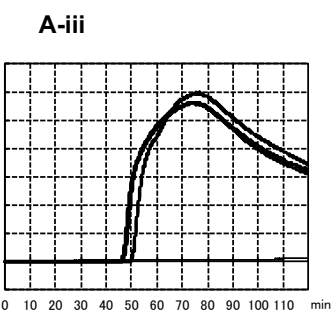

B-ii

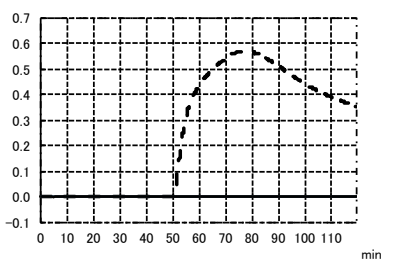

Fig. 3. Comparative amplification profiles of $A$. fumigatus wild type, and environmental or clinical azole resistant isolates with or without TR46 double or triple 46 bp promoter repeats in cyp51A gene by a newly developed LAMP primer sets. Dodded curve shows 


\section{the amplification by control strain (IFM 63432).}

A-i: DNA amplification profiles using 30 strains of $A$. fumigatus wild type. DNA amplification was not comfirmed in all wild type strains tested (30 strains). Dodded curve shows amplification by control strain.

A-ii: DNA amplification was comfirmed by five $\mathrm{TR}_{46}{ }^{2}$ strains (IFM63432, BE1-2, BE1-4, BE35, BE3-6) which have double 46 bp promoter repeats.

A-iii: DNA amplification was confirmed by three $\mathrm{TR}_{46}{ }^{3}$ strains (BE1-1, W1-4, W2-12-1) which have triple 46 bp promoter repeats.

B-I: DNA amplification was not confirmed by two $\mathrm{TR}_{34}{ }^{2}$ strains (IFM64460, IFM64733) which have duplicate $34 \mathrm{bp}$ promoter repeats with one mutations in the one coding region $(\mathrm{L} 98 \mathrm{H})$. Dodded line shows amplification by control strain.

B-ii: DNA amplification was not confirmed by one $\mathrm{TR}_{34}{ }^{2}$ strain (3-1-B) which has duplicate 34 bp promoter repeats with multi-mutations in the four coding regions (L98H/T289A/l364V/G448S). Dodded line shows amplification by control strain.

\section{Discussion}

Azole antifungals mainly inhibit the ergosterol biosynthetic pathway by targeting the cytochrome P450-dependent enzyme lanosterol 14-a-demethylase, encoded by cyp51A in molds. Resistance to this class of drugs in the major human pathogen A. fumigatus is emerging and reaching levels to prevent their clinical use (6). Advances in recent molecular genetic technologies such as real-time PCR have introduced various useful diagnostic assay methods into the fields in azole resistant mechanism analysis. The LAMP assay described here has advantages of high sensitivity and specificity, and also low costs and short 
amplification time. In addition, there have been no reports using LAMP techniques to study azole resistant mechanisms in $A$. fumigatus by $\mathrm{TR}_{46}$ gene.

Recently Yu Shan-Ling et al. ${ }^{15}$ reported a similar rapid technique to detect azole resistant strains due to amplification of a TR of a $34 \mathrm{bp}\left(\mathrm{TR}_{34}\right)$ and a $46 \mathrm{bp}\left(\mathrm{TR}_{46}\right)$ within the promoter region of cyp51A of $A$. fumigatus. However, here we used a newly designed TR46 LAMP primer sets which are different from those reported by Yu Shan-Ling et al. ${ }^{15}$.

There is a difference in MIC values between strains with $\mathrm{TR}_{46}$ and strains with $\mathrm{TR}_{34}{ }^{16}$. Thus, the importance of detecting $\mathrm{TR}_{46}$ lies in the fact that strains of $A$. fumigatus harboring $\mathrm{TR}_{46}$ are resistant to voriconazole, but not to itraconazole, while two $\mathrm{TR}_{34}$ strains (IFM64460: TR34/L98H and IFM64733: TR34/L98H) are highly resistant to voriconazole ${ }^{16}$ but not to itraconazole. Further detail drug susceptibility mechanism study against $\mathrm{TR}_{34}$ strain (3-1-B: TR34L98H/T289A/l364V/G448S) is of interest.

In this study we succeeded in the design of useful TR $\mathrm{R}_{46}$ LAMP primer sets to detect specifically a $\mathrm{TR}_{46}$ within the promoter regions of azole resistant $A$. fumigatus. The designed primer sets could differentiate azole resistant $\mathrm{TR}_{46}$ strains from that of $\mathrm{TR}_{34}$ strains. To our knowledge, this is a new and useful report of a detection method for one of the most prevalent cyp51A resistant gene $\mathrm{TR}_{46}$ in $A$. fumigatus azole resistant strains.

Recently, the strain consisting of the four repeats of $46 \mathrm{bp}$ of the promoter region was reported in the Netherlands $\left(\mathrm{TR}_{46}{ }^{4}\right)^{17}$. The LAMP primer we designed was able to detect both two copies of the TR46 tandem repeat and three copies of the TR 46 . Moreover, these amplification curves (as well as the starting point) were similar. The BIP (B1 + B2 complementary; Fig. 1) of the primer we designed is TR-specific. B1 is designed at the boundary where the repeat unit is inserted, and B2 is designed at the boundary between the repeat units. In addition, B3 is designed on a repeat unit. Based on the results of strains having double repeat and triple 
repeat, it was suggested that the primer used this time may be able to detect even if the number of repeats increases such as $\mathrm{TR}_{46}{ }^{4}$.

It is widely known that exposure to azole fungicides resulted in the emergence of azoleresistant strains with tandem repeats in the promoter region of cyp51A gene 8,9 . For this reason, epidemiological studies such as the incidence of azole-resistant strains in the environment are important. Many environmental and clinical isolates need to be screened to generate epidemiological data such as the frequency of detection of azole-resistant $A$. fumigatus. The method developed in this study would be an easy-to-use screening procedure.

Since the LAMP assay developed in the present study is one-step and rapid detection method, coupled with its high reliability and ease of use, it can be used for prompt specific detection of drug resistant genes due to $\mathrm{TR}_{46}$ in $A$. fumigatus in the clinical laboratory. Early detection of infections due to $\mathrm{TR}_{46}$ drug resistant strains in A. fumigatus, might be helpful to guide the early start of corrective and effective antifungal therapy.

\section{Methods}

\section{Aspergillus isolates and MIC determination by broth microdilution test}

Forty-one strains, including thirty-three from the clinical setting and eight environmental (plant bulbs) isolates ${ }^{18}$ of $A$. fumigatus were provided through the National Bio-Resource Project (NBRP), Japan (http://www.nbrp.jp/); source and drug susceptibility are shown in Table 1.

\section{DNA preparation and extraction}

Fungal strains were cultured on Sabouraud dextrose agar. Genomic DNA was extracted from 
over-night cultures of $A$. fumigatus mycelia by the urea-phenol method. Mycelia were mixed with $0.5 \mathrm{~mm}$ size glass beads, $0.5 \mathrm{ml}$ of $\mathrm{PCl}$ (phenol/chloroform/lsoamyl alcohol) solution and $0.5 \mathrm{ml}$ DNA extraction buffer (50 mM Tris-HCl, pH 8.0, 20 mM EDTA, $0.3 \mathrm{M} \mathrm{NaCl}, 0.5 \%$ SDS, $5 \mathrm{M}$ urea), and disrupted by Fast Prep FP100A (MP-Biomedicals, Santa Ana, USA). After centrifugation, the upper layer was transferred to a new tube and subjected to ethanol precipitation. The resulting DNA pellet was suspended in $100 \mu \mathrm{I}$ TE buffer. DNA concentration was determined by the methods described in our previous paper ${ }^{19}$.

All A. fumigatus strains were submitted to antifungal susceptibility tests according to the CLSI M38 protocol (https://clsi.org/standards/products/microbiology/documents/m38/), using Eiken Dried Plates (9DEF47, Eiken Chemical Co., Tokyo, Japan).

\section{LAMP-method}

LAMP was performed as described in our previous studies ${ }^{20}$. TR 46 LAMP primer was designed based on the target promoter region sequences of cyp51A gene of $A$. fumigatus, which includes tandem repeats in the promoter region containing $\mathrm{TR}_{46}$ mutant alleles. The sequence of the cyp51A gene were downloaded from NCBI Gen-Bank (https://www.ncbi.nlm.nih.gov/genebank). In total, a 184-bp nucleotide alignment (Fig. 1) was used for $\mathrm{TR}_{46}$ LAMP primer design by the protocol of the Eiken Company (Primer Explorer V5, Eiken Chemical Co. Ltd, Tokyo. Japan). LAMP primers are composed of six primers recognizing six distinct regions. The forward and backward inner primers, FIP/BIP, play crucial roles in the specificity of the assay. The outer primers, F3/B3, are composed of the fewer bases and are of a lower concentration than are FIP/BIP, initiating annealing of F3/B3 to the target in order to commence strand displacement. In addition to these four essential primers (FIP/BIP and F3/B3), the forward and backward loop primers (LF/LB) are used. The high specificity and rapidity of the present LAMP assay were achieved by applying 6 primers 
that target 6 regions of a DNA template, and 2 loop primers (LF, LB) to accelerate the reaction. LAMP reactions were performed with a Loopamp DNA amplification kit using reaction mixtures composed of 40 pmol each of primers FIP and BIP, 5 pmol each of primers F3 and B3, 20 pmol each of primers $L F$ and LB, $12.5 \mathrm{ml} \times 2$ reaction mixture, $1 \mu \mathrm{l}$ Bst DNA polymerase, $2 \mu \mathrm{L}$ DNA sample and distilled water up to a final volume of $25 \mu \mathrm{l}$ (Eiken Chemical Co., Ltd., Tokyo, Japan). The LAMP reactions were analyzed by a real-time turbidimeter (Loopamp EXIA; Eiken Chemical Co.) and were conducted at $63^{\circ} \mathrm{C}$, for $120 \mathrm{~min}$. The reaction mixtures were incubated at $61^{\circ} \mathrm{C}$ for 30 min (Realoop-30; Eiken Chemicals, Japan), and then heated at $80{ }^{\circ} \mathrm{C}$ for $2 \mathrm{~min}$ to terminate the reaction. Start of amplification of LAMP products at 30 to 50 minutes in the graph, suggested the positive reaction due to the presence of corresponding a $46 \mathrm{bp}$ tandem repeats of cyp51A gene is short. Since overall reaction can be obtained within 2 hours, prompt drug therapy can be deployed within a short time.

\section{Acknowledgements}

This study was supported by AMED under Grant Numbers JP20jm0110015 and by JICA through the collaborative research project Science and Technology Research Partnership for Sustainable Development (SATREPS), Japan.

\section{References}

1. Revie, N. M., Lyer, K. R,, Robbins, N. \& Cowen, L. E. Antifungal drug resistance: evolution, mechanisms and impact. Current Opin. Microbiol. 45, 70-76 (2018).

2. Chowdhary, A, Sharma. C., Hagen, F. \& Meis, J. F. Exploring azole antifungal drug resistance in Aspergillus fumigatus with special reference to resistance mechanisms. Future Microbiol. 9, 697-711 (2014).

3. Beer, K. D. et al. Multidrug-resistant Aspergillus fumigatus carrying mutations linked to 
environmental fungicide exposure - Three states, 2010-2017. MMWR Morb Mortal Wkly Rept. 67, 1064-1067 (2018).

4. Patterson, T. E. et al. Practice guidelines for the diagnosis and management of aspergillosis: 2016 update by the Infectious Diseases Society of America. Clin. Infect. Dis. 63, 438-442 (2016).

5. Hagiwara, D. et al. Epidemiological and genomic landscape of azole resistance mechanisms in Aspergillus fungi. Front. Microbiol. 7, 1382 doi: 103,389/fmicb.2016.01382.eCollection (2016).

6. Enserink, M. Infectious diseases: Farm fungicides linked to resistance in a human pathogen. Science 326(5957).1173. Doi: 10.1126/science,326.5957,1173 (2009).

7. Denning, D. W. et al. Chronic pulmonary aspergillosis: rationale and clinical guidelines for diagnosis and management. Eur. Respir. J. 47, 45-68 (2016).

8. Gsaller, F. et al. Sterol biosynthesis and azole tolerance is governed by the opposing actions of SrbA and the CCAT binding complex. PLOS Pathog. 12(7):e1005775. Doi: 10.1371/journal.ppt.1005775.eCollection (2016).

9. Nywening, A.V., Rybak, J. M., Rogers, P. D. \& Fortwendel, J. R. Mechanisms of triazole resistance in Aspergillus fumigatus. Environ. Microbiol. 22, 4924-4952 (2020).

10. Van Ingen, J. et al. Azole, polyene and echinocandin, MIC distribtions for wild-type, TR34/L98H and TR46/Y121F/T289A Aspergillus fumigatus isolates in the Netherlands. J. Antimicrob. Chemother. 70, 178-181 (2015).

11. Rößler, S. et al. Progressive dispersion of azole resistance in Aspergillus fumigatus: Fatal invasive aspergilosis in a patient with acute myeloid leukemia infected with an $A$. fumigatus strain with a cyp51A TR46 Y121FM1721 T289A allele. Antimicrob. Agents Chemother. 25, 61(8):e00270-17. Doi: 10.1128/AAC.00270-17(2017).

12. Rybak, J. M., Fortwendel, J. R., Rogers, P. D. Emerging threat of triazole-resistant 
Aspergillus fumigatus. J. Antimicrob. Chemother. 74, 835-842 (2019).

13. Sharpe, A. R., Lagrou, K., Meis, J. F., Chowdhary, A., Lockhart, S. R. \& Verweij, P. E. Triazole resistance surveillance in Aspergillus fumigatus. Med. Mycol. 56, S38-S92 (2018).

14. Inacio. J., Flores, O. \& Spencer-Matrins, I. Efficient identification of clinical relevant Candida yeast species by use of an assay combining panfungal loop-mediated isothermal DNA amplification with hybridization to species-specific oligonucleotide probes. J. Clin. Microbiol. 46, 713-729 (2008).

15. Ling, -S Y. et al. Rapid detection of azole-resistant Aspergillus fumigatus in clinical and environmental isolates by use of a lab-on-a-chip diagnostic system. J. Clin. Microbiol. 58, issue 11 e00843-20 ( 2020).

16. Dudakova, A. et al. Molecular tools for the detection and deduction of azole antifungal drug resistance phenotypes in Aspergillus species. Clin. Microbiol. Rev. 30, 1065-1091 (2017).

17.Zhang, J. et al. A novel environmental azole resistance mutation in Aspergillus fumigatus and a possible role of sexual reproduction in its emergence. mBio 27, 8(3):e00791-17. Doi: 10.1128/mBio.00791-17(2017).

18. Hagiwara, D. 2020. Isolation of azole-resistant Aspergillus fumigatus from imported plant bulbs in Japan and the effect of fungicide treatment. J. Pestic. Sci. 45, 147-150 (2020).

19. Trabasso, P. et al. Isolation and drug susceptibility of Candida parapsilosis sensu lato and other species of C. parapsilosis complex from patients with blood stream infections and proposal of a novel LAMP identification method for the species. Mycopathol. 179, 53-62 (2015).

20.de Souza, M. et al. Comparison of DNA microarray, loop-mediated isothermal amplification (LAMP) and real-time PCR with DNA sequencing for identification of Fusarium spp. obtained from patients with hematologic malignancies. Mycopathol. 182, 
625-632 (2017). 
Table Title

Table 1. Aspergillus fumigatus strains used in this experiment and their drug susceptibility profiles against itraconazole and voriconazole. 
Table 2. Sequence information of newly designed TR46-LAMP primer sets in the present experiment. 


\section{Figure legend}

Figure 1. Genetic information for the design of the LAMP primer sets.

A: Schematic illustration of cyp51A gene showing LAMP primer positions and corresponding sequences of TR46 bp promoter tandem repeat in comparion with those of wild type.

B: Primers F3, F2, F1, B1, B2 and B3 show primer sequence positions. Sequences of some primers

are complementary as shown in Table 2. See LAMP primer and methods which are shown in the refereces 19 and 20. 
Figure 2. Illustration of tandem repeat regions of cyp51A genes used in this experiment. A: Tandem repeat unit of promoter genes of $\mathrm{TR}_{34}$ and $\mathrm{TR}_{46}$.

B: Tandem repeat: $34 \mathrm{bp}$ (double) and $46 \mathrm{bp}$ (double or triple), and cyp51A gene associated point mutation place. 
Figure 3. Comparative amplification profiles of $A$. fumigatus wild type, and environmental or clinical azole resistant isolates with or without TR46 double or triple 46 bp promoter repeats in cyp51A gene by a newly developed LAMP primer sets. Dodded curve shows the amplification by control strain (IFM 63432).

A-i: DNA amplification profiles using 30 strains of $A$. fumigatus wild type. DNA amplification was not comfirmed in all wild type strains tested (30 strains). Dodded curve shows amplification by control strain.

A-ii: DNA amplification was comfirmed by five $\mathrm{TR}_{46}{ }^{2}$ strains (IFM63432, BE1-2, BE1-4, BE35, BE3-6) which have double 46 bp promoter repeats.

A-iii: DNA amplification was confirmed by three TR $46{ }^{3}$ strains (BE1-1, W1-4, W2-12-1) which have triple 46 bp promoter repeats.

B-I: DNA amplification was not confirmed by two $\mathrm{TR}_{34}{ }^{2}$ strains (IFM64460, IFM64733) which have duplicate $34 \mathrm{bp}$ promoter repeats with one mutations in the one coding region (L98H). Dodded line shows amplification by control strain.

B-ii: DNA amplification was not confirmed by one $\mathrm{TR}_{34}{ }^{2}$ strain (3-1-B) which has duplicate 34 bp promoter repeats with multi-mutations in the four coding regions (L98H/T289A/I364V/G448S). Dodded line shows amplification by control strain. 


\section{Figures}

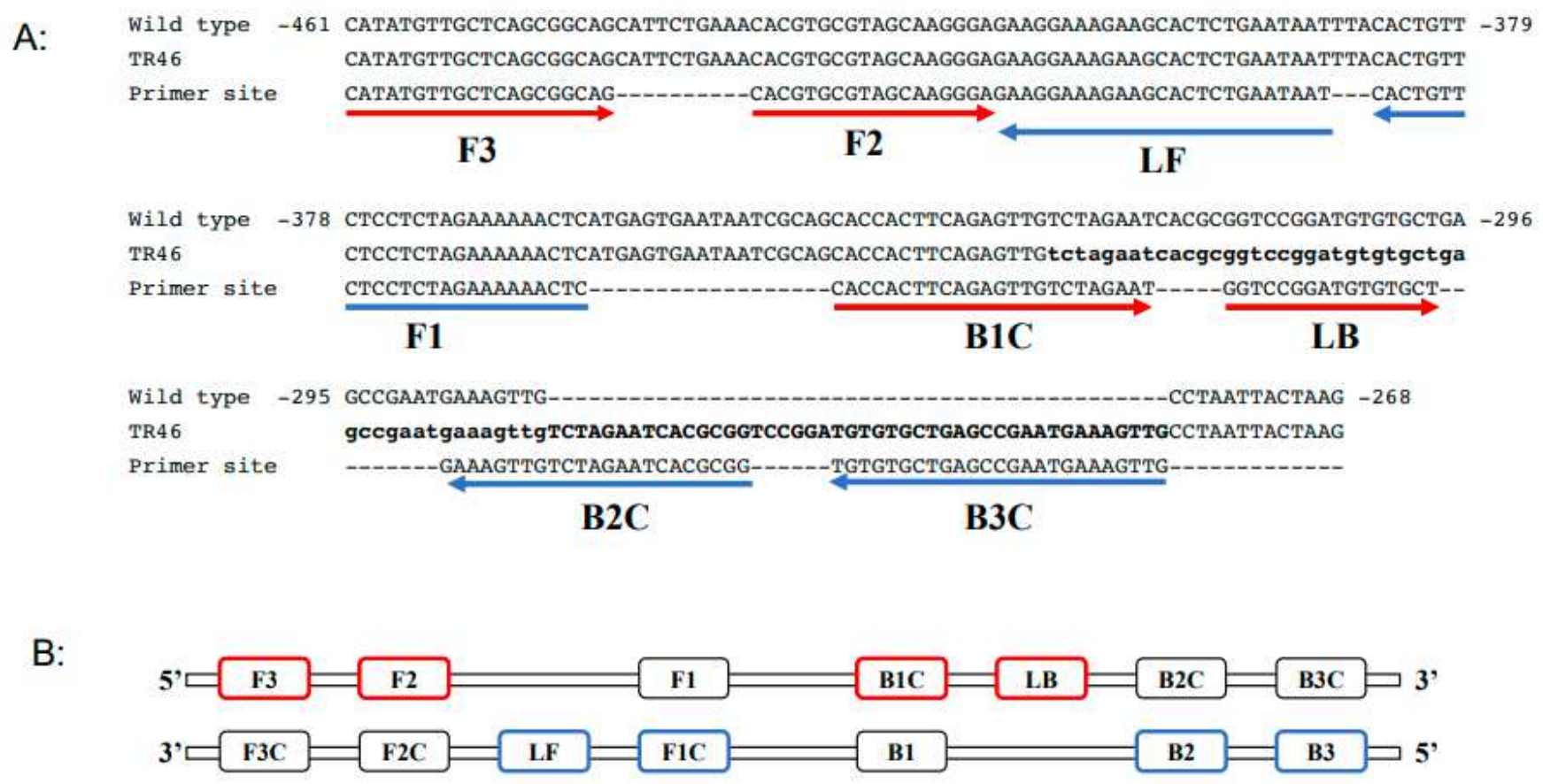

\section{Figure 1}

Please see the Manuscript PDF file for the complete figure caption 
A:

Repeat Unit of promoter region

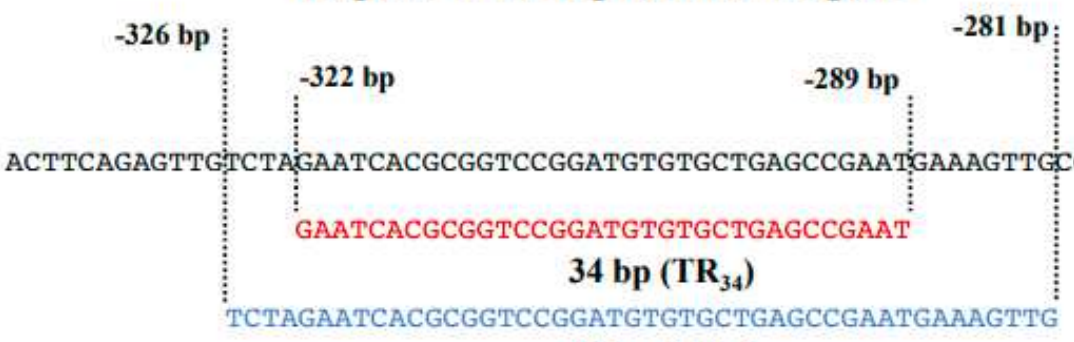

46 bp $\left(\mathrm{TR}_{46}\right)$

B:

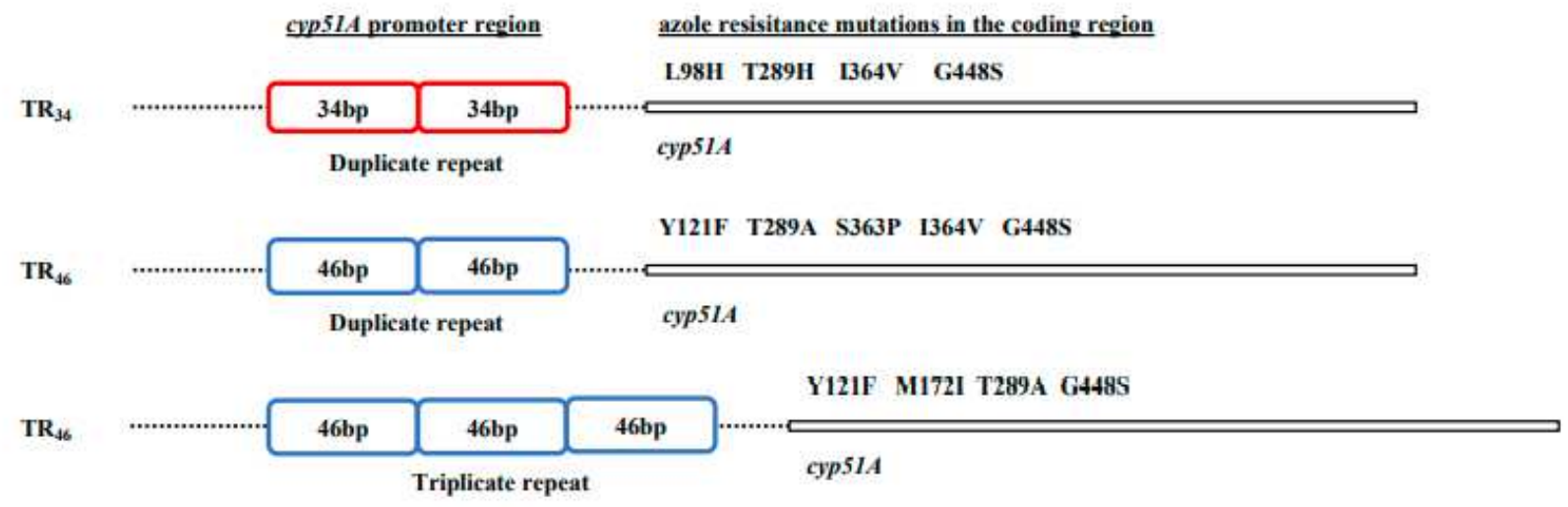

Figure 2

Please see the Manuscript PDF file for the complete figure caption

A

A-i

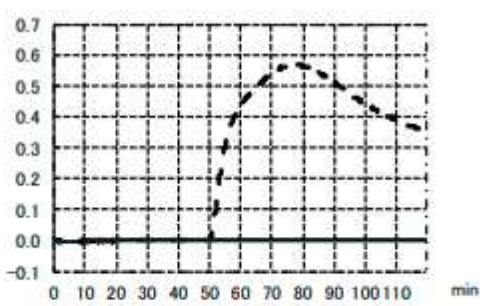

B

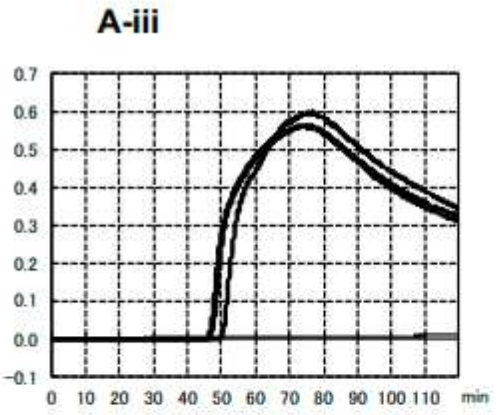

B-ii

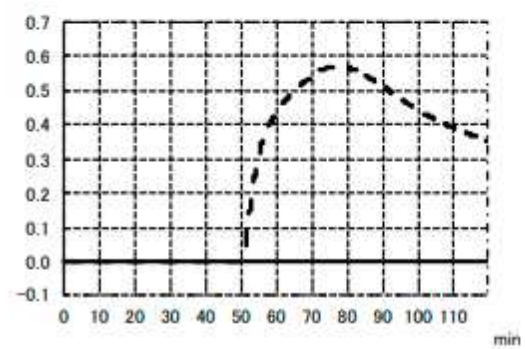

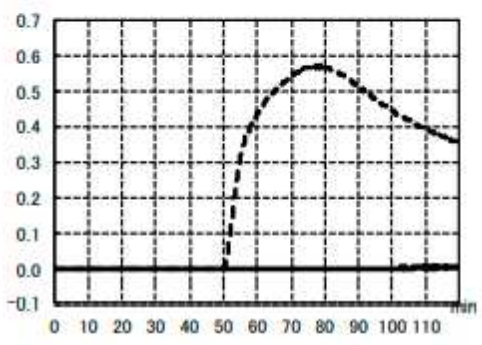


Please see the Manuscript PDF file for the complete figure caption 